\title{
Study on Fish Migration Caused by Sea Temperature Rise Based on Regression Analysis
}

\author{
Zihan Wu ${ }^{1}$, Zeqi Wang ${ }^{2}$, Kexin Guan ${ }^{3}$ \\ ${ }^{1}$ College of Mathematics, Tianjin Normal University, Tianjin, 300387, China \\ ${ }^{2}$ School of Public Administration, Huazhong Agricultural University, Wuhan, Hubei, 430000, China \\ ${ }^{3}$ College of Information Engineering, Sichuan Agricultural University, Ya'an, Sichuan, 625000, China
}

Keywords: Climate change, Time series, Sport fish movement model, Regression analysis model.

\begin{abstract}
Global warming has caused widespread concern around the world. The resulting changes in seawater temperature will seriously affect the migration of marine life, and as a result, some small fishing companies will have economic problems. We are honored to be hired as a consultant to analyze this and provide a feasible solution. We studied herring and mackerel in areas near Scotland. Firstly, we looked at the location information of the two types of fish school activity from 1970 to 2020, integrated them, and used the time series model and regression analysis model to predict the area and location of possible fish school activity in the next 50 years. The possible range of movement of the group, which helps the company to carry out timely stopping loss of the current fishing strategy and controlling the future economic regulation. In our prediction model, changes in sea surface temperature have a large impact on the migration behavior of fish schools. In order to prevent the timely fishing due to the influence of temperature, we give the best case, worst case, and most likely case after model prediction, and visualize the data by sorting, analyzing, and calculating. The process is more intuitive. Our forecasting model is affected by many factors, which will affect the economic benefits of the company. On the one hand, if changes are made to fishing vessels to keep the fish fresh and of high quality without land support, the cost in this case is too high; but on the other hand, if we transfer the company appropriate migration will not only allow the company to reduce costs, but also promote the exchange of regional cultures and jointly benefit. For the sake of climate change and economic benefits, we have given this plan. What is slightly inadequate is that the movement process of the animal group we considered is actually continuously changing in two-dimensional space and time. The process of establishing the model in three-dimensional space is not considered in the article. But the practicability and openness of the model are also its biggest advantages, and the sensitivity analysis in the process also makes the model more rigorous.
\end{abstract}

\section{Introduction}

Global warming is a climatic problem. It is due to the greenhouse effect that the energy absorbed and emitted by the geogas system is unbalanced. Energy is continuously accumulated in the geogas system, which leads to temperature rises and global warming. In order to adapt to the environment and continue to survive, schools of fish have begun to migrate. Many problems have emerged. Many fishing companies in coastal areas are facing new crises. This includes the impact of temperature changes on fish stocks. What kind of loss will the fish migration cause to the company's economy? In what way should we deal with such losses? We were hired as consultants to small fishing companies, and we needed to do some research on her most important herring and mackerel.

\section{Establishment and Solution of Model}

\subsection{0-year forecast}

Use MATLAB to batch cut the world sea surface temperature map that has been obtained, as shown in Figure 1 


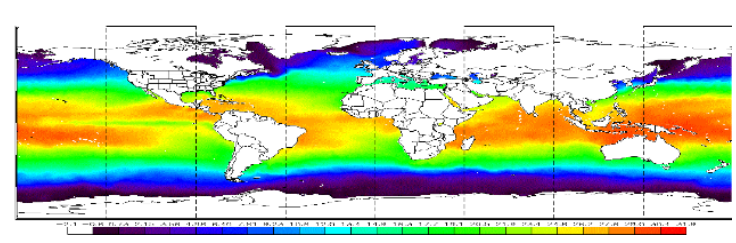

Figure 1 World Sea Surface Temperature Map

Obtain a specific sea surface temperature map in the Scottish waters, as shown in Figure 2.

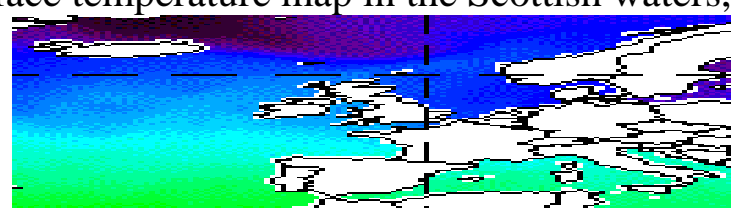

Figure 2 A specific sea surface temperature map in the Scottish waters

Obtain the value of relative temperature, replace this value with latitude and longitude, and get the latitude and longitude of two kinds of fish. Calculate the average latitude and longitude for each specific period of time, and calculate the relationship between longitude and latitude and time, and get the regression curve and regression equation. Take the next fifty years to get Figures 3 and 4 .

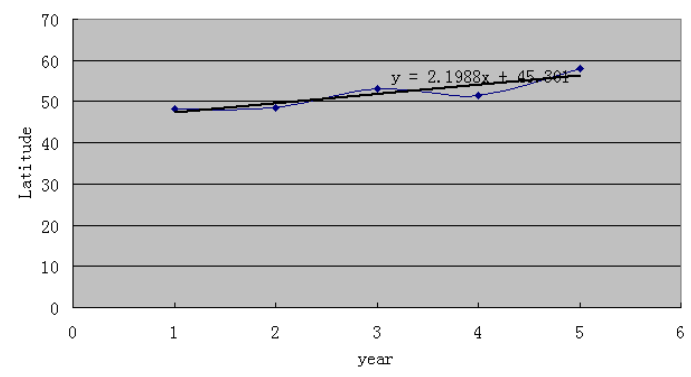

Figure 3 longitude

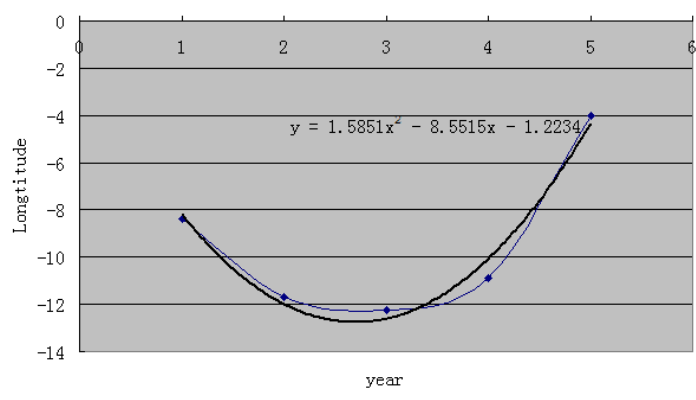

Figure 4 latitude and time

However, according to the actual situation, the fish's spanning ability is limited, and the longitude error in the data is large, so it can be inferred that the fish is still at the longitude $-4 \mathrm{E}-\mathrm{-}-12 \mathrm{E}$, and the latitude changes from 58.4938N-67.289N.

\subsection{Sport model of fish}

\subsubsection{Simplification of individual fish movement}

This article describes the case where a group of $\mathrm{N}$ individuals can be regarded as particles moving on the plane of the two-dimensional periodic boundary condition of $\mathrm{L}^{*} \mathrm{~L}$, and the speed of each individual fish movement is the same at each moment.

\subsubsection{Definition of individual fish}

Each individual fish is a self-driven individual with the same shape and size and a certain perception ability. The range it can perceive is a circular area of radius $\mathrm{R}$ with its center of mass as the 
dot, and it can perceive all the dynamic information of other individual fishes in this area (including the speed and direction, and whether there is an alarm signal, etc.) .

\subsubsection{Description of motion rules}

(1) Rule of inertia. When an individual fish receives a signal that needs to change its movement, the direction in which the fish swims cannot change immediately. At this time, it shows an inertia effect

(2) Close to the rule, in order not to leave the school of fish, you need to be as close to the center of the neighbor as possible.

(3) Alignment rules. In order to maintain the coherence of fish movement, each individual fish should try to be consistent with the direction of its neighbors.

(4) Avoidance rules. In order to maintain the consistency of fish movement, individual fish movements should avoid collisions as much as possible.

\subsubsection{The establishment of a motion model}

Assume that each individual fish has the same speed at time $t$, and the direction of the speed of the movement is arbitrary or random, and the position coordinates are known in a given plane. The establishment of an individual fish's motion model is to study the direction and position of the individual fish at the next moment $\mathrm{t}+1$ ( 1 represents a time step) at the coordinate plane. The four rules in 3 all have a certain effect on changing the swimming direction of the fish at the next moment, so in this paper, the average direction of these four directions is taken as the direction of the fish's next moment. Take individual fish A1 to study its movement. Because the direction is the angle with the horizontal direction, it is only necessary to average the angles between the above four directions and the horizontal direction, that is, the swimming direction of the fish at the next moment. Formulated as:

$$
P_{t+1}=P_{1 t}+P_{2 t}+P_{3 t}+P_{4 t}
$$

Where $p_{t+1}$ is the moving direction of the individual fish in the next time step period, $p_{1 t}$ is the swimming direction of A1 in period t, $p_{2 t}$ is the direction from the current individual to the average position of the neighbor at period t, $p_{3 t}$ is the average direction of the neighbor, and $p_{4 t}$ is less than the average of the collision distance's neighbors to the current individual direction.

Considering that the rules have different influences on fish, individual fishes, as the self-driven decision-makers, have different priorities when they make decisions, so they need to weight each direction and take a weighted average. The formula is transformed into:

$$
P_{t+1}=\lambda_{1} P_{1 t}+\lambda_{2} P_{2 t}+\lambda_{3} P_{3 t}+\lambda_{4} P_{4 t}
$$

Where $\lambda_{1}+\lambda_{2}+\lambda_{3}+\lambda_{4}=1$. The specific implementation methods of the four directions represented by the four rules are given below:

(1) Implementation of the inertia rule: $P_{1 t}$ is the swimming direction of A1 in period $\mathrm{t}$, and the movement direction during this week is determined by the movement direction at the previous moment, that is, $P_{1 t}=P_{t-1}$.

(2) Implementation of the proximity rule: Each individual has the characteristic of moving closer to the center of the neighbor, and the center of the neighbor is the average value of the positions of the individuals in the observation range. Assume that the current position of A1 is $D_{0}\left(x_{0}, y_{0}\right)$, and $D_{i}\left(x_{i}, y_{i}\right)$ is the position of each current neighbor. Then, the average position of the neighbor $\bar{D}(\bar{x}, \bar{y})=\frac{\sum D_{i}\left(x_{i}, y_{i}\right)}{|N|}(i \in N)$, and $P_{2 t}$ is the direction from the current individual to the average position of the neighbor at period t:

$$
P_{2 t}=\arctan \frac{\bar{y}-y_{0}}{\bar{x}-x_{0}}
$$


(3) Implementation of alignment rules: An individual will swim in the same direction as its neighbors. The formula is expressed as:

$$
P_{3 t}=\frac{\sum P_{i}}{|N|}\left(\left|D_{0} D_{i}\right| \leq R\right)
$$

Where $P_{i}$ is the direction of each neighbor, $\mathrm{N}$ is the number of neighbors, and $P_{3 t}$ is the average direction of the neighbors.

(4) Implementation of the collision avoidance rule: When an individual is too close to its neighbor (the distance is less than the collision distance), it should be avoided automatically. The formula is expressed as:

$$
P_{4 t}=\sum \arctan \frac{y_{0}-y_{i}}{x_{0}-x_{i}} / M \quad\left(\left(\left|D_{0} D_{i}\right| \leq r\right)\right)
$$

Where $P_{4 t}$ is the average of the neighbors less than the collision distance to the current individual direction, and $\mathrm{M}$ is the number of neighbors that are less than the collision distance.

Finally, according to the above calculation, it can be obtained that the position iteration rule of the individual A1 is:

$$
\left\{\begin{array}{l}
x_{t+1}=x_{t}+v \cdot \cos P_{t} \\
y_{t+1}=y_{t}+v \cdot \sin P_{t}
\end{array}\right.
$$

First, this article predicts the results based on the analysis of the model:

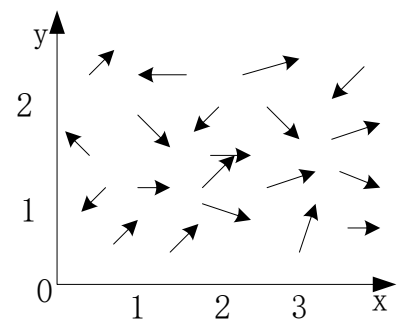

(a) the initial state of a school of fish

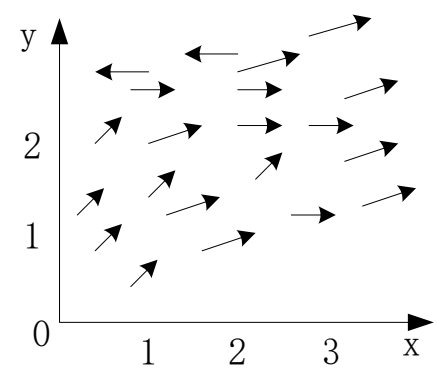

(b) the clustering effect of school of fish after $\mathrm{t} s$

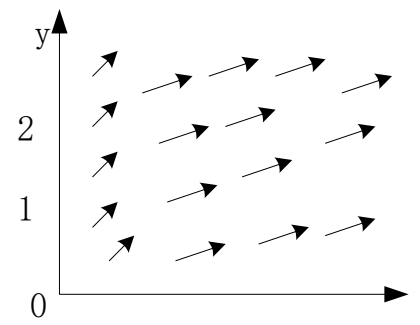

(c) cluster effect of fish school after running $t+n \mathrm{~s}$

Figure 5 Fish movement effect 


\subsection{Simulation of fish motion model(Sensitivity analysis)}

This paper uses Matlab software to simulate the results of the model. When the model starts to run, a certain number of individuals are randomly distributed in the flat environment. Each individual has its own state attributes, that is, its initial state is random. During the running of the model, individuals constantly move their positions and interact with the environment and other individuals. In $P_{t+1}=\lambda_{1} P_{1 t}+\lambda_{2} P_{2 t}+\lambda_{3} P_{3 t}+\lambda_{3} P_{3 t}$, the parameter is set to $\lambda_{1}=0.5, \lambda_{2}=0.2, \lambda_{3}=0.2, \lambda_{4}=0.1$; and the object attributes are set: the number of fish is 100 , the speed is 0.5 , the sensing range is 5 units, and the minimum distance for collision is $5 / 5=1$ unit. And the simulation results are obtained.

\subsection{Analysis results of fish movement model}

(1) The initial state of the individual in the school of fish, that is, the direction of movement and position coordinates are randomly given. It can be seen in the figure that the school of fish does not have a regular group movement behavior.

(2) After a period of exercise, according to the motion model of individual fish, each individual fish follows the same swimming rules. With the continuous change of state parameters, the school of fish starts to slowly gather and tend to a consistent state of movement.

(3) When the model runs for a long time, the cluster movement behavior of the school of fish emerges. Based on their respective motion models, the individual fish schools achieve continuous matching from local to overall through continuous coordination, and finally form a unified and synchronized operating structure.

(4) According to the overall sexual behavior of the school of fish and the changes in the swimming distance and speed of the fish obtained, the final best case is 20.0606 hours, the worst case is 14.5137 hours, and the most likely case is 17.2872 hours.

\subsection{Evaluation of two business strategies}

Discussed in two situations:

Moving company:

$\mathrm{P} 1=\mathrm{A}(\mathrm{t})-\mathrm{n} 1{ }^{*} \mathrm{~s}^{*} \mathrm{o} 1-\mathrm{B}(\mathrm{t})-\mathrm{S}(\mathrm{t})$

Do not move company:

$\mathrm{P} 2=\mathrm{A}(\mathrm{t})-\mathrm{n} 2 * \mathrm{~s} * \mathrm{o} 2-\mathrm{S}(\mathrm{t})$

It is assumed that the movement pattern of other species is the same as that of the studied fish school, so the fishing volume is not significantly different from the previous one, and it will not be affected by predators and predators, the environment, and water quality. Because many data cannot find specific values, and these values are related to the age, they are expressed as time-related expressions; a country's gross domestic product can reflect the country's prices, wages (both with GDP) Directly proportional); so we find the change in the British Gross Domestic Product from 1970 to 2016, and make it every ten years to solve the average, and get the regression equation: $\mathrm{G}=6531 \mathrm{t}-5035.6$

After consulting the data, we can see that a large ship has a capacity of 5 tons and a small ship has a capacity of 0.5 tons. In the same case, the ratio of the number of large ships and boats is $1: 10$. The boat consumes 7.5 liters per 100km of operation, and the price of each liter of gasoline is 6.6 yuan. Suppose there were 10 large ships, and there were also 100 small ships. By

$$
\begin{aligned}
& \mathrm{P} 1=A(\mathrm{t})-\mathrm{n} 1{ }^{*} \mathrm{~s}^{*} \mathrm{o} 1-\mathrm{B}(\mathrm{t})-\mathrm{S}(\mathrm{t}) \\
& \mathrm{P} 2=\mathrm{A}(\mathrm{t})-\mathrm{n} 2{ }^{*} \mathrm{~s}{ }^{*} \mathrm{o} 2-\mathrm{S}(\mathrm{t})
\end{aligned}
$$

The only difference is that the consumption of moving a large ship once and the consumption of a small ship that does not move the company. It is better to move the company once every ten years by analyzing the existing data, and the farther you move to the north, the farther away from the national center Rent is cheap, so we think it's better to choose to move the company every ten years. 


\section{The charm of regional culture}

According to the first question, it can be found that the fish survival area will hover between $-4 \mathrm{E}$ to $-12 \mathrm{E}$, and due to the increase in global temperature and the optimal temperature of fish, they will need to migrate northward in the future. From 2010 — The average latitude of the school of fish in 2019 is about 57.88704N, which is about Aberdeen in the United Kingdom. It already belongs to the northern part of the UK. If the temperature continues to rise, the school of fish will go all the way north. From the first question regression prediction, it can be concluded that it is from $58.4938 \mathrm{~N}$ to 67.289N. The countries and regions to be passed through are Britain (Homeland), Iceland, Faroe Islands, Denmark, Norway. It will be affected by national borders and violate the territorial sea of other countries, which will cause unnecessary disputes. During the relocation process, companies will be subject to the management of foreign company policies in different countries and cultural differences between different countries. These factors will increase the cost, so we will explain these factors

\subsection{Iceland and Faroe Islands}

Iceland is an important fishing country in Northern Europe, because the region belongs to the extreme north, and its fishing output is relatively low compared to other countries. Global warming has led to a northward shift in fish activity, which provides a great opportunity for Iceland to increase fisheries production. Take mackerel as an example. In 2005, the annual output of Icelandic mackerel fishing was only 5651 tons, and with global warming, Icelandic mackerel fishing output began to increase sharply year by year. In 2009, the output surged to 115,000 tons. It increased to 130 thousand tons in 2010 and 147,000 tons in 2011, which is about three times higher than the fishing quota set by the European Union. At the same time, the Faroe Islands also set a quota of 85,000 tons of mackerel by itself in 2010, with an output of 115,000 tons in 2011 [2]. Setting up a new company in Iceland will be difficult. Moreover, Iceland is dissatisfied with the current allocation method for mackerel fishing quotas, and Norway resolutely opposes the requirements of Iceland and other countries to adjust the mackerel fishing quotas significantly. If foreign companies enter the homeland, Iceland's own companies will have fewer fishing limits. Considers setting up a company in Iceland relatively unadvisable. The most important thing is that the "Cod War" broke out between Britain and Iceland in history, so I don't think Iceland will agree to Britain's entry into the domestic market, and British companies will not choose their "enemies" as economic partners.

\subsection{EU and Norway}

Norway and the EU member states are important fishing countries in the North Atlantic. Norway's annual marine fisheries production is about 2 million tons, ranking 15th in the world. Among them, the largest species are cod, salmon and mackerel, and 30\% of the North Atlantic mackerel fishing quota is owned by Norway. In 2010, Norway's aquatic products exports amounted to US $\$ 8.8$ billion, ranking second only to China in the world [3]. And I think Scotland can take advantage of this to build a factory in Norway. Norway has a large fishing quota for fish and there

will be exports every year, so we think Norway should not reject foreign companies.

\subsection{Greenland, Denmark may become a new member of the mackerel dispute}

Also due to climate change, Greenland's ice sheets and glaciers have melted a lot. In the summer of 2012, the number of mackerel schools in the Greenland waters increased significantly. Greenland showed great interest in this, and fishermen regarded it as a "pie from the sky." Greenland and the Faroe Islands previously shared quotas, and the fishing industry in Greenland was dominated by Arctic red shrimp and tadpoles, and the fishery species were relatively monotonous. Greenland has been hoping to increase fishing species and promote diversification of Greenland fisheries species. Global warming and the northward shift of fish activity have provided a good opportunity for Greenland, which is dominated by fisheries, to develop its economy and change its domestic fishery structure. In addition to fishing production, Greenland also needs to invest in new mackerel processing plants locally and build larger processing mother ships to serve as catchment receiving 
stations on fishing grounds. We think that a country's fishing industry is booming, and he will not refuse the influx of foreign resources, so I think we can also set up factories in Greenland.

\section{References}

[1] Xiu Xianjie, Yang Zhenzhen, Lin Jiatao, Zhang Ping, Xu Hongze, Zhang Yongle, Chen Guang. Effects of herring mackerel migration on North Sea fisheries J. China Science and Technology Information, 2020(08):118-120.

[2] Yao cuiluan, George N.Somero. effects of ocean warming on marine organisms J. science bulletin, 2015,60(09):805-816.

[3] Tian Mingwei. Modeling and Simulation of Complex Group Behavior of Fish School in Three-dimensional Space D. Yanshan University, 2013.

[4] Zhao Jian, Zeng Jianchao. Modeling and simulation of fish swarm behavior J. Journal of Taiyuan University of Science and Technology, 2009, 30(01): 31-35.

[5] Ma Xinliang. Global warming endangers marine life J. Fujian Environment, 2001(04): 39. 